\title{
Modified atomic force microscopy cantilever design to facilitate access of higher modes of oscillation
}

\author{
S. Sadewasser, ${ }^{\text {a) }}$ G. Villanueva, and J. A. Plaza \\ Instituto de Microelectrónica de Barcelona, CNM-CSIC, Campus UAB, 08193 Bellaterra (Barcelona), Spain
}

(Received 15 March 2006; accepted 12 June 2006; published online 18 July 2006)

\begin{abstract}
The detection of higher modes of oscillation in atomic force microscopy can provide additional information on sample properties. However, the limited bandwidth of the photodiode in the beam deflection detection technique often limits the detectable frequency range. We present a novel cantilever design, which allows lowering the frequency of higher modes of oscillation, maintaining the frequency of the fundamental resonance. Simulations by finite element methods show that the ratio between the second mode and the fundamental resonance frequency can be adjusted between 2 and 10, compared to 6.3 for the regular rectangular cantilever design. Even stronger changes are observed for the third oscillation mode, corresponding to a torsional cantilever oscillation. Cantilevers with modified geometry were fabricated using silicon micromachining processes and subsequently tested, confirming the results from the simulation. () 2006 American Institute of Physics. [DOI: 10.1063/1.2219738]
\end{abstract}

\section{INTRODUCTION}

The atomic force microscope (AFM) has developed into an important tool for materials characterization over the past two decades. ${ }^{1}$ The dynamic force microscope or noncontact AFM (NC-AFM) is currently applied in a large variety of fields including semiconductor science, biology, and chemistry. It employs an oscillating cantilever holding a tip at its free end. ${ }^{2}$ Changes in the oscillation frequency or amplitude are monitored or compensated for measuring the topography of sample surfaces with nanometer resolution, even down to atomic resolution. ${ }^{3}$ Recently it has been proposed that the detection of higher harmonic oscillations can provide information on additional physical surface or material properties. ${ }^{4}$ The use of the second oscillation mode has been used in Kelvin probe force microscopy (KPFM) for the simultaneous determination of the samples contact potential. ${ }^{5-7}$ An ac-bias is applied to the sample at the frequency of the second mode of oscillation of the cantilever. The electrostatic force excites an additional cantilever oscillation, which is then minimized by applying a compensating dc bias, which corresponds to the contact potential. This technique, however, limits the cantilevers to be used; this is because the photodiode of the beam deflection detection system of commercial AFMs provides an upper bandwidth of usually about $500 \mathrm{kHz}$. The resonance frequencies of the different modes of oscillation for a rectangular shaped cantilever are given by ${ }^{8}$

$$
f_{n}=\frac{\lambda_{n}^{2}}{2 \pi} \frac{t}{l^{2}} \sqrt{\frac{E}{12 \rho}}
$$

where $E$ is Young's modulus, $\rho$ the density of the cantilever material, $l$ and $t$ are the length and the thickness of the cantilever, respectively, and $\lambda_{n}$ a factor depending on the mode

\footnotetext{
${ }^{a}$ Present address: Hahn-Meitner Institut, Glienicker Strasse 100, 14109 Berlin, Germany.
}

of oscillation. For the first three modes, $\lambda_{1}=1.88, \lambda_{2}=4.69$, and $\lambda_{3}=7.86 .{ }^{8}$ This results in a relation between the frequency of the second mode of oscillation and the fundamental mode of $f_{2} / f_{1} \approx 6.3$. Thus, limiting $f_{2}$ by the bandwidth of the photodiode $(\sim 500 \mathrm{kHz})$ limits the fundamental frequency to $f_{1} \approx 80 \mathrm{kHz}$. Nevertheless, higher fundamental frequencies are desired for reasons of stability when imaging the topography.

Another wide field of application of cantilevers is its use as sensors for, i.e., gases, molecules, ${ }^{9}$ or mass. ${ }^{10}$ Here, either the mass uptake or the surface stress modifies the resonance frequency. Due to the higher quality factor of higher resonance modes these are more sensitive and their application should increase the experimental resolution. ${ }^{11}$

With the goal to access information about the tip-sample interaction contained in induced oscillation of higher harmonics, a modified cantilever geometry has been used to shift a specific higher harmonic of the fundamental oscillation (i.e., $n \cdot f_{1}$ ) to coincide with a higher eigenmode of oscillation, thereby increasing the sensitivity of this specific harmonic and easy its detection. ${ }^{12}$

We present here a novel design for the geometry of cantilevers for AFM which allows to freely adjust the ratio between the resonance frequencies of the second and third modes of oscillation with respect to the fundamental mode. The design was optimized using finite element simulation and silicon micromachining processes were used for fabrication of such cantilevers. Experimental frequency spectra confirm the simulation results.

\section{SIMULATION}

We investigated the effect of changing the geometry of the cantilever on the fundamental resonance frequency $f_{1}$, the second $\left(f_{2}\right)$, and third $\left(f_{3}\right)$ modes of oscillation and their ratios $f_{2} / f_{1}$ and $f_{3} / f_{1}$, respectively. The change in geometry 

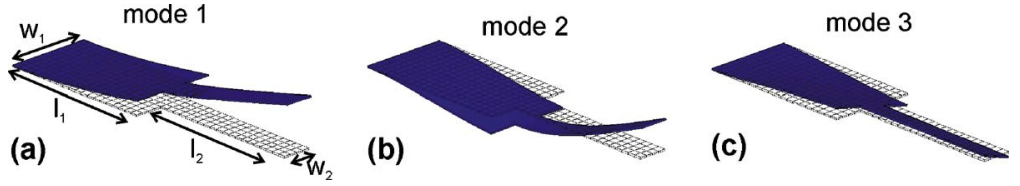

FIG. 1. FEM simulation of (a) the fundamental resonance mode, (b) the second, and (c) the third modes of oscillation of a cantilever with a modified geometry, consisting of a wider part of width $w_{1}$ and length $l_{1}$ anchored to the chip and a narrower part of width $w_{2}$ and length $l_{2}$ at the free end of the cantilever. consists of a variation of the width of the cantilever as a function of the distance from the chip supporting the cantilever. The simplest approach to this variation in geometry is to divide the cantilever in one part with a width $w_{1}$ and length $l_{1}$ anchored to the chip and a second part of width $w_{2}$ and length $l_{2}$ defining the free end of the cantilever. The thickness $t$ is assumed to be constant throughout the length of the cantilever. The geometry is shown and the parameters are defined in Fig. 1(a). The finite element method (FEM) was applied (program ANSYS) to predict the effect of various combinations of $l_{1}, l_{2}, w_{1}$, and $w_{2}$ on the resonance frequencies and their ratios. The geometric shape of the fundamental (resonance frequency $\left.f_{1}\right)$, the second $\left(f_{2}\right)$, and the third $\left(f_{3}\right)$ mode of oscillation are shown in Figs. 1(a)-1(c), respectively. As a result of the simulation we obtained the frequencies $f_{1}, f_{2}$, and $f_{3}$, respectively.

\section{A. Results for the second resonance mode of oscillation}

Figure 2(a) shows the dependence of the ratio $f_{2} / f_{1}$ for a variation of the lengths $l_{1}$ and $l_{2}$ of the wider and the narrower part, respectively, maintaining the total length $l=l_{1}+l_{2}$ constant at $200 \mu \mathrm{m}$. The result is presented for
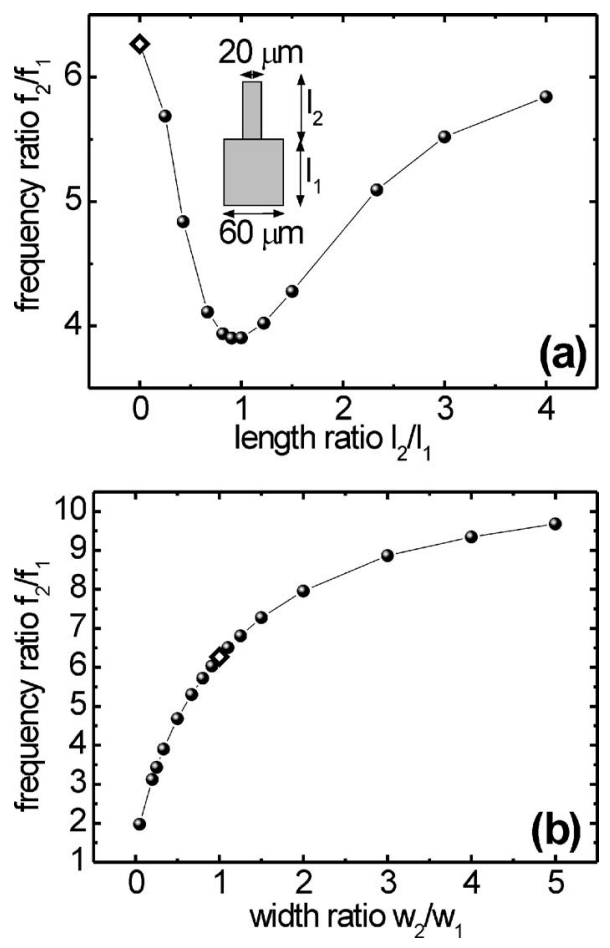

FIG. 2. Results of the FEM simulation of cantilevers with a modified geometry. (a) Ratio of the resonance frequencies of the second $\left(f_{2}\right)$ and the fundamental $\left(f_{1}\right)$ mode of oscillation for a variation of the lengths of a wider anchored part $l_{1}$ and a narrower free end $l_{2}$ of the cantilever. A minimum in $f_{2} / f_{1}$ is observed for $l_{1}=l_{2}$. (b) Resonance frequency ratio $f_{2} / f_{1}$ for a variation of the width ratio between the free and the anchored part of a cantilever consisting of two equally long parts $\left(l_{1}=l_{2}=100 \mu \mathrm{m}\right)$. $w_{1}=60 \mu \mathrm{m}$ and $w_{2}=20 \mu \mathrm{m}$. A minimum in the ratio $f_{2} / f_{1}$ is seen for $l_{2} / l_{1}=1$, meaning that the lowest frequency $f_{2}$ with respect to the fundamental resonance frequency $f_{1}$ is obtained when both parts, the wider and the narrower, have the same length. In this case the ratio is $f_{2} / f_{1} \approx 3.9$, which compares to the ratio of $f_{2} / f_{1} \approx 6.3$ for the rectangular geometry (see the diamond in Fig. 2). It is also seen that the ratio $f_{2} / f_{1}$ is always lower than 6.3 of the rectangular geometry, meaning that for the fixed width relation, a length variation does not allow to further separate the frequencies of the resonance modes. In comparison, the effect for varying the widths $w_{1}$ and $w_{2}$ is shown in Fig. 2(b). Here both parts are assumed to be equally long, $l_{1}=l_{2}=100 \mu \mathrm{m}$ and the narrower part is anchored for $w_{2} / w_{1}>1$ and free for $w_{2} / w_{1}<1$. The width of the narrower part is fixed to $w_{2}=20 \mu \mathrm{m}$. It is seen that $f_{2} / f_{1}<6.3$ when the wider part is anchored. The ratio can be as low as 2 for $w_{2} / w_{1}=0.05$, corresponding to a width $w_{1}$ $=400 \mu \mathrm{m}$. However, in practice such a wide cantilever will have a strong impact on the mechanical behavior of the cantilever; for example, the quality factor $Q$ would be reduced due to the extreme width. On the other hand, $f_{2} / f_{1}>6.3$ when the narrower part of the cantilever is anchored. This means that the resonance frequencies are shifted apart; this can be advantageous to avoid possible cross talk between the modes.

\section{B. Results for the third oscillation mode}

As higher resonance modes promise higher sensitivity in experimental applications, ${ }^{11}$ we also studied the effect of the modified cantilever geometry on the third resonance mode, i.e., the torsional bending, as shown in Fig. 1(c). For the configuration of varying the width $w_{1}$ and $w_{2}$ of a cantilever composed of two equally long $\left(l_{1}=l_{2}=100 \mu \mathrm{m}\right)$ and equally thick $(t=1 \mu \mathrm{m})$ parts, the ratio $f_{3} / f_{1}$ of the third resonance mode and the fundamental resonance mode was studied by finite element simulations. The results in Fig. 3 show a quite different dependence in comparison to the results for the ratio $f_{2} / f_{1}$. The ratio $f_{3} / f_{1}$ shows a maximum at $\sim 17.5$ for the case of the rectangular geometry and varies between this value and 2 in the simulated widths range. Thus, it results that the ratio $f_{3} / f_{1}$ can only be reduced with respect to the value for the rectangular cantilever geometry, in contrast to the ratio $f_{2} / f_{1}$, which can be increased and decreased, as shown in Fig. 2(b). This difference could be a result of the selected geometry consisting of two parts with different width. Possibly a different arrangement with several sections of different widths might provide the possibility to increase the ratio $f_{3} / f_{1}$.

\section{Effect of a variation in cantilever thickness}

The previously described cases of a modified geometry considered a cantilever with a width variation. We also stud- 


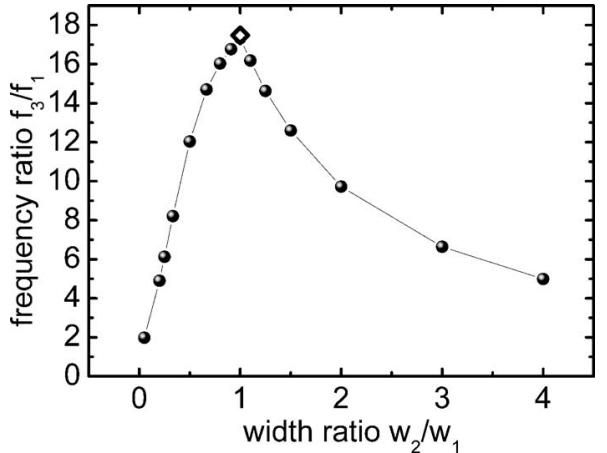

FIG. 3. Results of the FEM simulation of cantilevers with a modified geometry. Ratio of the resonance frequencies $f_{3}$ and $f_{1}$ of the third (torsional) and the fundamental modes of oscillation, respectively, for a variation of the width of a cantilever consisting of two equally long parts $\left(l_{1}=l_{2}=100 \mu \mathrm{m}\right)$. The ratio $f_{3} / f_{1}$ can be adjusted between 2 and 17.5, which is the maximum obtained for a rectangular geometry (open diamond).

ied the effect of varying the thickness of a cantilever with a homogeneous width. The cantilever consists of two parts, the anchored part with thickness $t_{1}$ and the free end with thickness $t_{2}$. Both parts are equally long $\left(l_{1}=l_{2}=100 \mu \mathrm{m}\right)$ and have the same width of $40 \mu \mathrm{m}$ (see inset of Fig. 4). The results of the finite element simulation for the ratio of the frequencies of the second and fundamental modes of oscillation, $f_{2} / f_{1}$, as a function of the thickness ratio $t_{2} / t_{1}$ are shown in Fig. 4. The dependence shows a minimum with a value of $\sim 3$ for $t_{2} / t_{1}=0.375$ and then increases towards above 12 for the range of thicknesses studied. Thus, also a variation of the thickness allows the variation of the frequency ratio $f_{2} / f_{1}$ over a wide range, including the possibilities of bringing the frequencies of the first and second modes closer together or to separate them further, in comparison to the rectangular geometry. The different tendency for the thickness variation, when comparing to the results of the width variation is due to the fact that for a large difference in thickness (when $t_{2} / t_{1}$ is either very small or very large) the thicker part of the cantilever does only show a very small bending, whereas the thinner part is responsible for most of the bending. This is also expressed in the higher modes; for example, in the third mode, the torsional bending of the thin-

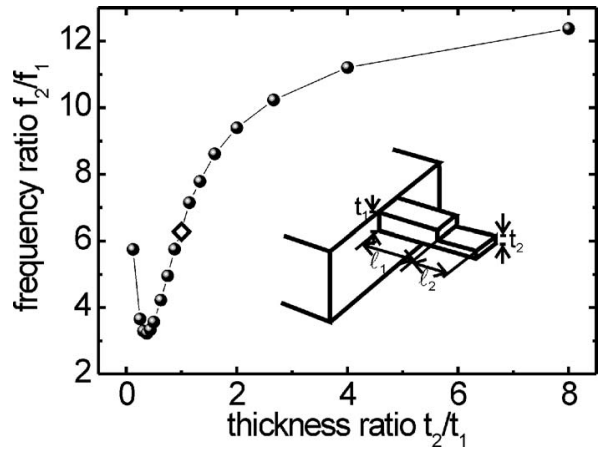

FIG. 4. Results of the FEM simulation of cantilevers with a modified geometry. Ratio of the resonance frequencies $f_{2}$ and $f_{1}$ of the second and the fundamental modes of oscillation, respectively, for a variation of the thickness of a cantilever consisting of two equally long parts $\left(l_{1}=l_{2}=100 \mu \mathrm{m}\right)$. The ratio $f_{2} / f_{1}$ can be adjusted between 3 and above 12 and shows a minimum for $h_{2} / h_{1}=0.375$. The open diamond shows the ratio for the rectangular geometry. a)

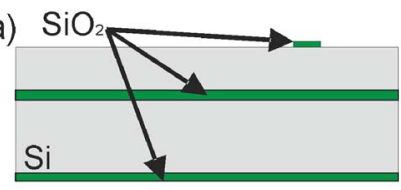

d)

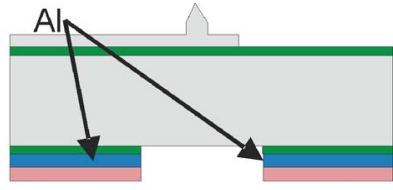

b)

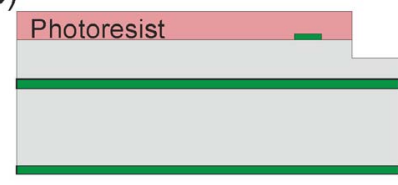

e)

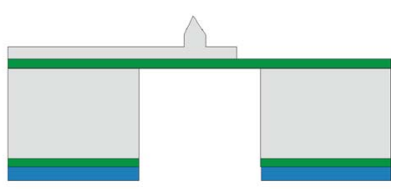

c)

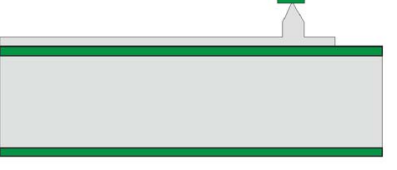

f)

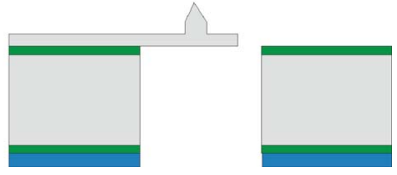

FIG. 5. Schematic drawings of the fabrication process of the cantilevers with modified geometry. See text for details.

ner part of the cantilever dominates and is responsible for almost all the oscillation.

\section{FABRICATION}

The fabrication is based on a 4 in. $450-\mu$ m-thick silicon on insulator (SOI) wafer with a $15-\mu \mathrm{m}$ thick top silicon layer and a $1-\mu \mathrm{m}$-thick buried silicon oxide layer. First a silicon oxide layer is thermally grown and patterned on the front side [Fig. 5(a)], to be used as mask material for the tip formation. In the next step a photolithographic process is performed, in which the photoresist is used as mask for a partial silicon etching of about $2 \mu \mathrm{m}$ [Fig. 5(b)]; this etching defines the cantilevers. After photoresist removal two dry processes in an inductively coupled plasma (ICP) reactor are carried out. The first one is a nonvertical profile process that shapes the apex of the tip [Fig. 5(c)]. The second one shapes the column of the tip using the vertical profile that can be obtained with ICP reactors. It has to be noticed that the second etching also defines the thickness of the cantilevers. Subsequently, an aluminium layer is deposited and patterned at the backside of the wafer [Fig. 5(d)]. This layer is used as mask material for the three-dimensional (3D) machining of the whole chip by a deep reactive ion etching (DRIE) process from the backside [Fig. 5(e)]. The etch stops at the buried silicon oxide layer of the SOI wafer. Finally, this oxide is removed to release the cantilevers [Fig. 5(f)]. At this point, the AFM probes are held in the wafer by two small hinges that can be broken by manual cleaving.

\section{EXPERIMENTAL RESULTS}

A typical result of the fabrication of cantilevers with modified geometry is shown in the scanning electron microscopy (SEM) image in Fig. 6. The cantilever was designed to have a width of the wider, anchored part of the cantilever of $w_{1}=60 \mu \mathrm{m}$ and of the narrower free end of the cantilever of $w_{2}=20 \mu \mathrm{m}$. Both parts have the same length $l_{1}=l_{2}$ $=100 \mu \mathrm{m}$, as was found in the simulations to provide the lowest ratio $f_{2} / f_{1}$. However, during the fabrication process some lateral etching of the silicon cantilever occurred such 


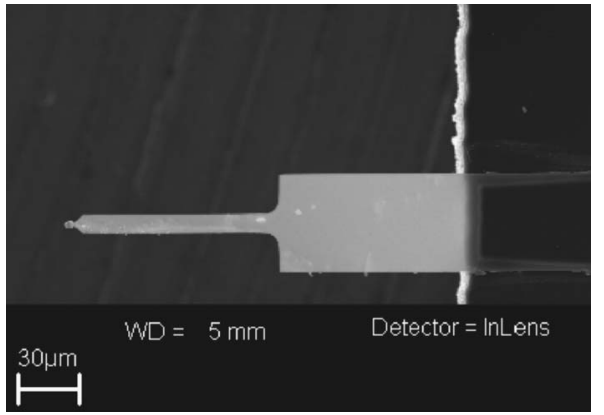

FIG. 6. Scanning electron microscopy image of a cantilever with modified geometry for reduction of the frequency ratio $f_{2} / f_{1}$. The frequency spectrum of this cantilever is shown in Fig. 7.

that the dimensions of the cantilever are reduced by $\sim 5 \mu \mathrm{m}$ from each side, resulting in a width of the wider anchored part of $w_{1} \sim 50 \mu \mathrm{m}$ and of the narrower free end of the cantilever of $w_{2} \sim 10 \mu \mathrm{m}$. The frequency spectrum of the cantilevers was measured in a commercial AFM (Nanotec, Spain) in ambient air using an excitation amplitude of $0.3 \mathrm{~V}$ and a gain of 30. The amplitude versus frequency plot of the cantilever shown in Fig. 6 is presented in Fig. 7 and shows two resonance peaks for the fundamental resonance at $f_{1}$ $\approx 40.7 \mathrm{kHz}$ and for the second mode of oscillation at $f_{2}$ $\approx 136.6 \mathrm{kHz}$. The peaks are fairly sharp, however, some noise in the frequency range between the resonances can be observed. The quality factor of the fundamental oscillation mode can be determined from the width of the resonance peak to $Q=f / \Delta f \approx 45$. This is a reasonable value for cantilevers subject to damping in ambient air. The frequency ratio is indeed lower than for the regular rectangular cantilever geometry and is found to be $f_{2} / f_{1} \approx 3.4$. This deviates slightly from the simulation result seen in Fig. 2; however, introducing the different width of the actual cantilever as found from the SEM image in Fig. 6, the experimentally found frequency ratio is in good agreement with the one from simulation $f_{2} / f_{1} \approx 3$.2. Due to the bandwidth of the photodiode of the used AFM system, the third oscillation mode (torsional mode, $f_{3} \approx 500 \mathrm{kHz}$ ) was not accessible in the experiment.

To obtain information on the reproducibility and reliability of the fabrication process we examined a set of up to eight cantilevers of three different geometries. Detailed information of the geometries is given in Table I. Cantilever geometries $A$ and $B$ have a total length of $200 \mu \mathrm{m}$ with a

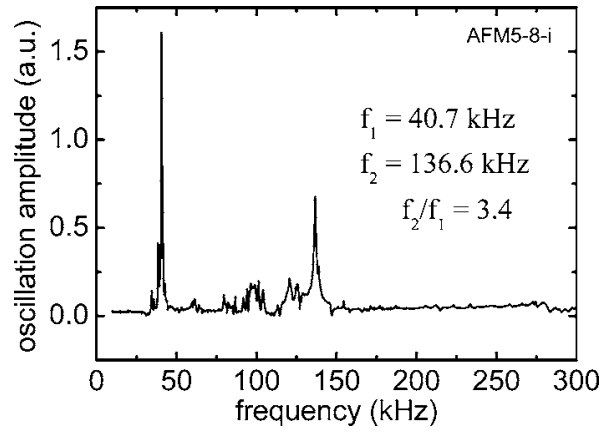

FIG. 7. Frequency spectrum of the cantilever shown in Fig. 6. The fundamental resonance mode is at $f_{1}=40.7 \mathrm{kHz}$ and the second mode of oscillation is at $f_{2}=136.6 \mathrm{kHz}$, resulting in a ratio of $f_{2} / f_{1}=3.4$.

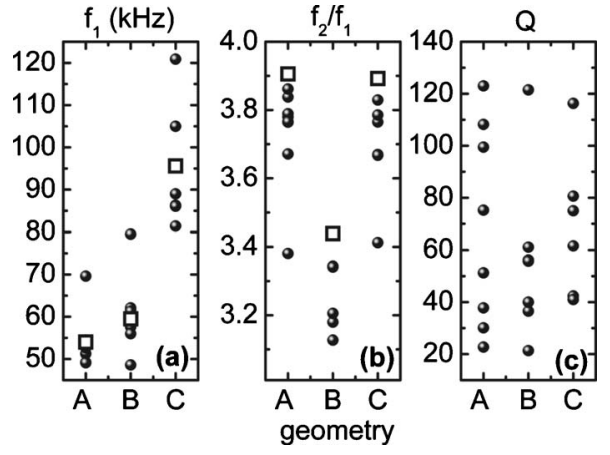

FIG. 8. Evaluation of a set of cantilevers with different geometries $A, B$, and $C$ according to Table I. The experimental values for (a) the fundamental resonance frequency $f_{1}$ and (b) the ratio between the frequencies of the second and fundamental modes of oscillation, $f_{2} / f_{1}$, are compared to the values obtained from the simulation (open squares). The experimentally determined quality factor is shown in (c).

different width of the anchored part, whereas geometry $C$ has a total length of only $150 \mu \mathrm{m}$ with the same widths as geometry $A$. The wider and the narrower part of the cantilever are equally long in all three cases $\left(l_{1}=l_{2}\right)$. We measured the frequency spectrum of a set of eight cantilevers for each geometry, and analyzed its fundamental resonance frequency $f_{1}$, the ratio between the second mode and the fundamental frequency $f_{2} / f_{1}$ and the quality factor $Q$. Figure 8 gives an overview of these parameters for the various cantilevers, separated for the different geometries. A good agreement is seen between the fundamental resonance frequencies from experiment and simulation. There is a quite large variation within the experimental values of $f_{1}$. This is most likely due to thickness variations: in the fabrication process the thickness of the cantilever is defined only by the etch rate (with a $5 \%$ cross wafer nonuniformity) of the top Si layer which also has a thickness nonuniformity up to $1 \mu \mathrm{m}$. Lateral variations in the etch rate and thus the resulting cantilever thickness are quite likely, since the different chips were taken randomly from the wafer. Due to the linear dependence of the resonance frequency on the thickness, we can estimate the thickness variation which would account for the experimental variation in $f_{1}$, finding $0.80 \mu \mathrm{m}<t<1.35 \mu \mathrm{m}$.

The simulated ratio $f_{2} / f_{1}$ is slightly higher than the experimentally found values for all three geometries. This corresponds to the finding for the cantilever discussed above and presented in Figs. 6 and 7. The lateral etching of the structures modifies the ratio of the widths $w_{2}$ and $w_{1}$ and therefore changes the simulated frequency ratio $f_{2} / f_{1}$ towards lower values.

The experimentally found quality factor $Q$ for the various cantilevers in Fig. 8 varies between 20 and 120. This range is typically found for oscillating cantilevers and is due to the damping in ambient air.

Recently, the sensitivity of the optical beam detection for oscillations at higher eigenmodes has been studied for AFM cantilevers with regular geometry; ${ }^{13,14}$ positioning the spot of the detection laser to the free end of the cantilever was found to be optimal. Despite the fact that the mode shape for the presented cantilever geometry should be slightly different, these findings should be transferable to the presented geom- 
TABLE I. Parameters defining the geometry of the different cantilevers examined in this study. All cantilevers have a homogeneous thickness $t \sim 1 \mu \mathrm{m}$ and two differently wide parts of equal length. The widths $w_{1}$ of the anchored part and $w_{2}$ of the free cantilever end, as well as the total length $l_{\mathrm{tot}}=l_{1}+l_{2}$ are given in the table. The simulated values for the fundamental frequency $f_{1}$, the ratio to the second mode $f_{2} / f_{1}$, and the ratio to the third mode $f_{3} / f_{1}$ are also given.

\begin{tabular}{ccccccc}
\hline \hline Geometry & $\begin{array}{c}l_{\text {tot }} \\
(\mu \mathrm{m})\end{array}$ & $\begin{array}{c}w_{1} \\
(\mu \mathrm{m})\end{array}$ & $\begin{array}{c}w_{2} \\
(\mu \mathrm{m})\end{array}$ & $\begin{array}{c}f_{1} \\
(\mathrm{kHz})\end{array}$ & $f_{2} / f_{1}$ & $f_{3} / f_{1}$ \\
\hline A & 200 & 60 & 20 & 54.0 & 3.9 & 8.2 \\
B & 200 & 80 & 20 & 59.5 & 3.4 & 6.1 \\
$\mathrm{C}$ & 150 & 60 & 20 & 95.6 & 3.9 & 6.5 \\
\hline \hline
\end{tabular}

etry, especially, when considering that the dimensions of the free cantilever end and its oscillation behavior are similar to those of a cantilever with the regular geometry.

\section{DISCUSSION}

We have proposed a novel geometry for cantilevers to be used in AFM when higher modes of oscillation are to be monitored to obtain information on additional sample properties or when the cantilevers are used as sensors for gases, molecules, or mass detection. This modification from the regular rectangular geometry consists in a wider anchored part and a narrower free end. Simulations showed that the frequencies of the second and third oscillation mode with respect to the fundamental oscillation mode can be adjusted over a wide range, either in order to lower the ratios $f_{2} / f_{1}$ and $f_{3} / f_{1}$ for better accessibility or to shift them apart for minimization of possible cross talk. Simulation results were used to optimize the geometry and subsequently fabricate cantilevers using $\mathrm{Si}$ micromachining processes. Frequency spectra confirm the results of the simulations. Thus, we have successfully developed a new geometry for AFM cantilevers which will allow access to additional sample properties on the nanoscale.

\section{ACKNOWLEDGMENT}

The authors gratefully acknowledge financial support from the Spanish government through the MINAHE (TIC2002-04280-C03-02) and MINAHE 2 projects (TEC2005-07996-CO2-01).

${ }^{1}$ G. Binnig, C. F. Quate, and Ch. Gerber, Phys. Rev. Lett. 56, 930 (1986). ${ }^{2}$ Y. Martin, C. C. Williams, and H. K. Wickramasinghe, J. Appl. Phys. 61, 4723 (1987).

${ }^{3}$ F. J. Giessibl, Science 267, 68 (1995).

${ }^{4}$ R. W. Stark, Nanotechnology 15, 347 (2004).

${ }^{5}$ A. Kikukawa, S. Hosaka, and R. Imura, Appl. Phys. Lett. 66, 3510 (1995).

${ }^{6}$ Ch. Sommerhalter, Th. W. Matthes, Th. Glatzel, A. Jäger-Waldau, and M. Ch. Lux-Steiner, Appl. Phys. Lett. 75, 286 (1999).

${ }^{7}$ S. Sadewasser, Th. Glatzel, M. Rusu, A. Jäger-Waldau, and M. Ch. LuxSteiner, Appl. Phys. Lett. 80, 2979 (2002).

${ }^{8}$ H.-J. Butt and M. Jaschke, Nanotechnology 6, 1 (1995).

${ }^{9}$ H. Jensenius, J. Thaysen, A. A. Rasmussen, L. H. Veje, O. Hansen, and A. Boisen, Appl. Phys. Lett. 76, 2615 (2000).

${ }^{10}$ Z. J. Davis, G. Abadal, O. Kuhn, O. Hansen, F. Grey, and A. Boisen, J. Vac. Sci. Technol. B 18, 612 (2000).

${ }^{11}$ L. B. Sharos, A. Raman, S. Crittenden, and R. Reifenberger, Appl. Phys. Lett. 84, 4638 (2004).

${ }^{12}$ O. Sahin, G. Yaralioglu, R. Grow, S. F. Zappe, A. Atalar, C. Quate, and O. Solgaard, Sens. Actuators, A 114, 183 (2004).

${ }^{13}$ R. W. Stark, Rev. Sci. Instrum. 75, 5053 (2004).

${ }^{14}$ T. E. Schäffer and H. Fuchs, J. Appl. Phys. 97, 083524 (2005). 